\title{
PADRÃO DE DISTRIBUIÇÃO DE ESPÉCIES DE SCARABAEINAE (COLEOPTERA, SCARABAEIDAE) EM MATA ATLÂNTICA
}

\author{
$\underline{\text { Gesica Regina Gomes da Silva }}{ }^{1}$; Priscila Paixão Lopes ${ }^{2}$
}

1. Bolsista PIBIC/FAPESB, Graduanda em Licenciatura em Ciências Biológicas, Universidade Estadual de Feira de Santana, e-mail: gesicaregina@gmail.com

2. Orientador, Departamento de Biologia, Universidade Estadual de Feira de Santana, e-mail: pplc29@ gmail.com

PALAVRAS-CHAVE: Scarabaeinae; Mata Atlântica; Distribuição.

\section{INTRODUÇÃO}

A comunidade Scarabaeinae apresenta uma diversidade com cerca de 7000 espécies conhecidas, abundantemente encontradas em ambientes tropicais, distribuídas em todo o mundo. Compreende os besouros chamados de rola-bostas devido aos seus hábitos comportamentais de formação e excrementos animais. Dados atualizados reportam, que até 2011 teriam sido descritas um total de 700 espécies em todo pais, sendo que uma pequena porcentagem deste número já é conhecida nas diversas coleções entomológicas. A Mata Atlântica apresenta uma maior disponibilidade de microhabitats, clima e alimentos, o que explica a maior abundância e riqueza de Scarabaeinae nessas áreas. Diante disto é importante investigar os besouros, em relação a sua presença e diversidade, buscando um aprofundamento sobre esta comunidade na Mata Atlântica, que em algumas áreas pelo país são desenvolvidos trabalhos neste espaço, porem muitos não são publicados e divulgados, gerando assim uma lacuna na gama de informações que se possui sobre os escarabeídeos. Com isto buscamos a sistematização de informações a cerca dos Scarabaeinae que ocorrem na faixa de Mata Atlântica no país.

\section{MATERIAL E MÉTODOS OU METODOLOGIA}

Os dados analisados correspondem tanto a dados de literatura, em que foram oferecidas informações sobre comunidades de Scarabaeinae, quanto dados ainda não publicados obtidos pela equipe de pesquisa de Scarabaeinae do Laboratório de Entomologia (LENT), da UEFS. Os dados de literatura foram obtidos na internet, a partir de buscas pelo Portal Periódicos da CAPES, utilizando os termos de busca "Scarabaeidae", "Scarabaeinae", "Mata Atlântica", "Floresta Atlântica" de forma combinada, e termos equivalentes em inglês. A mesma busca foi realizada no Google Acadêmico. Os artigos encontrados foram baixados e analisados quanto à disponibilidade de dados de ocorrência e/ou estrutura de comunidade (abundâncias). Foram obtidas informações de caracterização fitofisionômica (florestas fechadas, preservadas, perturbadas ou manchas urbanas) e coordenadas geográficas. A partir dos dados foram construídas duas tabelas com ocorrência de espécies, sendo a primeira com os dados completos segundo o apresentado pelos autores (incluindo morfoespécies, para comparações de diversidade, e outra desconsiderando as morfoespécies (com sp, aff., gr., prox.). Analisando os dados tabelados, para descrever as morfoespécies, foi realizada a compatibilização e descrição das áreas que apresentavam trabalhos com desenvolvimentos semelhantes e/ ou mesma localização, como também a compatibilização e checagem das espécies com ocorrência ampla na maioria dos trabalhos. Para comparação das riquezas dos vários estudos 
realizados ao longo da Mata Atlântica, usamos todos os dados, incluindo morfoespécies, considerando apenas presenças/ausências. Foram calculadas as riquezas estimadas para cada área (Chao1) e comparadas quanto à efetividade de coleta determinada pela proporção entre a riqueza e a riqueza estimada para a comunidade (PAST, HAMMER et al., 2001). A variação de riqueza foi correlacionada com variáveis geográficas, determinando possíveis efeitos latitudinais, representado em uma forma gráfica.

\section{RESULTADOS E/OU DISCUSSÃO}

Foram analisados 27 artigos científicos e nove trabalhos de conclusão, sendo uma monografia, cinco dissertações de mestrado, e três teses. Aos dados de distribuição foram agregados dados de pesquisas da equipe de Scarabaeidologia do Laboratório de Entomologia da UEFS de coletas realizadas em vários pontos no domínio Mata Atlântica no estado da Bahia, num total de 13 áreas. Os trabalhos analisados foram distintos em termos de objetivos. apresentavam finalidades para especificar algumas delimitações dentro da comunidade, como: variações sazonais e espaciais, especificidades e descrição sobre os hábitos alimentares, efeitos da degradação sobre a comunidade, estabilidade das mesmas em áreas de mata, assim como a presença em determinadas áreas, descrição e distribuição de guildas tróficas e principalmente a influencia do habitat e suas características sobre a comunidade. Apresentado muitas finalidades o trabalhos desenvolvidos, consequentemente juntos tornam-se uma gama de informações importantes, aprofundando ainda mais sobre esta área. Em função desses objetivos distintos, apresentaram métodos de coleta não homogêneos, como iscagem diferencial no caso de uso de pitfalls e diferentes esforços amostrais. Uma grande oferta de iscas possibilita a maior procura por parte dos besouros e aumenta a riqueza de espécies em determinado espaço amostral. Em relação à localização das comunidades ao longo da faixa de Mata Atlântica, os trabalhos abarcaram áreas desde a Paraíba até o Rio Grande do Sul, sem registros para os estados do Rio de Janeiro (sudeste), Rio grande do Norte e Ceará (nordeste). A distribuição concentrada de trabalhos refletiu a distribuição de pesquisadores nos estados de Paraíba, Pernambuco, Bahia, Minas Gerais, Santa Catarina e Rio Grande do Sul. Algumas áreas apresentaram repetição de representação por terem sido repetidamente amostradas em diferentes estudos. As áreas escolhidas para desenvolvimento dos trabalhos variaram entre matas secundárias, semidecíduas, florestas latifoliadas, contínuas, formações arbóreas, agroflorestas e áreas próximas a pastagens. Todos os trabalhos apresentavam listas com as espécies coletadas durante o tempo de pesquisa, apontando a diversidade encontrada na áreas coletadas; sendo hábitat propício para a comunidade de Scarabaeinae, a Mata Atlântica é uma das áreas do mundo que apresenta os maiores índices de diversidade (Hernández \& Endres 2011), tanto em fauna como flora. Dentre as espécies registradas, 28 apresentaram mais de 10 registros e nove delas apresentaram distribuição com grande amplitude geográfica desde o nordeste até o sul do país (Tabela 1). 
Tabela 1. Ocorrências das espécies de Scarabaeinae mais frequentemente registradas em estudos de Mata Atlântica, por estado. Apenas espécies com 10 ou mais registros.

\begin{tabular}{|c|c|c|c|c|c|c|c|c|c|c|c|c|}
\hline \multirow[b]{2}{*}{ Espécie } & \multicolumn{10}{|c|}{ Estados } & \multirow{2}{*}{$\begin{array}{l}\text { Registro } \\
\mathrm{s}\end{array}$} & \multirow{2}{*}{$\begin{array}{l}\mathrm{N}^{\circ} \text { de } \\
\text { estados }\end{array}$} \\
\hline & $\begin{array}{l}\mathrm{P} \\
\mathrm{B}\end{array}$ & $\begin{array}{l}\mathrm{P} \\
\mathrm{E}\end{array}$ & $\begin{array}{l}\mathrm{A} \\
\mathrm{L}\end{array}$ & $\begin{array}{l}\text { B } \\
\text { A }\end{array}$ & $\begin{array}{l}M \\
G\end{array}$ & $\begin{array}{l}\mathrm{E} \\
\mathrm{S}\end{array}$ & SP & $\begin{array}{l}\mathrm{P} \\
\mathrm{R}\end{array}$ & $\begin{array}{l}\mathrm{S} \\
\mathrm{C}\end{array}$ & $\begin{array}{l}\mathrm{R} \\
\mathrm{S}\end{array}$ & & \\
\hline Deltochilum brasiliense & & & & $\mathrm{X}$ & $\mathrm{X}$ & & $\mathrm{X}$ & $\mathrm{X}$ & $\mathrm{X}$ & $\mathrm{X}$ & 34 & 6 \\
\hline Dichotomius mormon & & & $\mathrm{X}$ & $X$ & $\mathrm{X}$ & & $\mathrm{X}$ & $\mathrm{X}$ & & & 31 & 5 \\
\hline Coprophanaeus dardanus & & $\mathrm{X}$ & $X$ & $\mathrm{X}$ & $\mathrm{X}$ & $\mathrm{X}$ & $\mathrm{X}$ & & $\mathrm{X}$ & & 28 & 7 \\
\hline Eurysternus cyanescens & & & & $\mathrm{X}$ & $\mathrm{X}$ & & $\mathrm{X}$ & & $\mathrm{X}$ & & 23 & 4 \\
\hline Eurysternus paralellus & & & & & $\mathrm{X}$ & & $\mathrm{X}$ & $\mathrm{X}$ & $\mathrm{X}$ & $\mathrm{X}$ & 22 & 5 \\
\hline Canthon nigripenne & $\mathrm{X}$ & $\mathrm{X}$ & $\mathrm{X}$ & $\mathrm{X}$ & & & & & & & 21 & 4 \\
\hline Phanaeus splendidulus & & & & $\mathrm{X}$ & $\mathrm{X}$ & & $\mathrm{X}$ & $\mathrm{X}$ & $\mathrm{X}$ & $\mathrm{X}$ & 21 & 6 \\
\hline Eurysternus caribaeus & & $\mathrm{X}$ & & $\mathrm{X}$ & $\mathrm{X}$ & $\mathrm{X}$ & & $\mathrm{X}$ & $\mathrm{X}$ & $\mathrm{X}$ & 20 & 7 \\
\hline Ontherus Azteca & & & & $\mathrm{X}$ & $\mathrm{X}$ & & $\mathrm{X}$ & $\mathrm{X}$ & $\mathrm{X}$ & $\mathrm{X}$ & 19 & 6 \\
\hline Coprophanaeus bellicosus & & & $\mathrm{X}$ & $\mathrm{X}$ & $\mathrm{X}$ & & $\mathrm{X}$ & & & & 18 & 4 \\
\hline Dichotomius depressicolis & & $\mathrm{X}$ & $\mathrm{X}$ & $\mathrm{X}$ & $\mathrm{X}$ & & $\mathrm{X}$ & $\mathrm{X}$ & & & 18 & 6 \\
\hline Deltochilum furcatum & & & & & $\mathrm{X}$ & & $\mathrm{X}$ & $\mathrm{X}$ & $\mathrm{X}$ & & 17 & 4 \\
\hline Coprophanaeus saphirinus & & & & & $\mathrm{X}$ & & $\mathrm{X}$ & $\mathrm{X}$ & $\mathrm{X}$ & $\mathrm{X}$ & 16 & 5 \\
\hline Deltochilum morbillosum & $\mathrm{X}$ & & & & $\mathrm{X}$ & & $\mathrm{X}$ & $\mathrm{X}$ & $\mathrm{X}$ & $\mathrm{X}$ & 15 & 6 \\
\hline Sylvicanthon foveiventre & & & & $\mathrm{X}$ & $\mathrm{X}$ & & $\mathrm{X}$ & & & & 15 & 3 \\
\hline Chalcocopris hespera & & & & & $\mathrm{X}$ & $\mathrm{X}$ & $\mathrm{X}$ & & & & 13 & 3 \\
\hline Deltochilum rubripenne & & & & & $\mathrm{X}$ & & $\mathrm{X}$ & $\mathrm{X}$ & $\mathrm{X}$ & $\mathrm{X}$ & 13 & 5 \\
\hline Canthidium aterrimum & & & & $\mathrm{X}$ & $\mathrm{X}$ & & $\mathrm{X}$ & & & & 12 & 3 \\
\hline Canthon chalybaeus & & $\mathrm{X}$ & & & $\mathrm{X}$ & & & $\mathrm{X}$ & $\mathrm{X}$ & $\mathrm{X}$ & 12 & 5 \\
\hline Eurysternus hirtellus & $\mathrm{X}$ & & $\mathrm{X}$ & & $\mathrm{X}$ & & $\mathrm{X}$ & $\mathrm{X}$ & & $\mathrm{X}$ & 12 & 6 \\
\hline Canthonella silphoides & & & & $\mathrm{X}$ & & & & & & & 11 & 1 \\
\hline Dichotomius assifer & & & & & $\mathrm{X}$ & & $\mathrm{X}$ & $\mathrm{X}$ & & $\mathrm{X}$ & 11 & 3 \\
\hline Dichotomius iannuzziae & & & & $\mathrm{X}$ & & & & & & & 11 & 1 \\
\hline Dichotomius nisus & $\mathrm{X}$ & $\mathrm{X}$ & $\mathrm{X}$ & $\mathrm{X}$ & & & & $\mathrm{X}$ & & $\mathrm{X}$ & 11 & 6 \\
\hline Dichotomius quadrinodosus & & & & & $\mathrm{X}$ & & $\mathrm{X}$ & & $\mathrm{X}$ & & 11 & 3 \\
\hline Onthophagus catharinensis & & & & & & & $\mathrm{X}$ & $\mathrm{X}$ & $\mathrm{X}$ & $\mathrm{X}$ & 11 & 4 \\
\hline Coprophanaeus jasius & $\mathrm{X}$ & & & & $\mathrm{X}$ & & & $\mathrm{X}$ & & & 10 & 3 \\
\hline Deltochilum irroratum & $\mathrm{X}$ & $\mathrm{X}$ & $\mathrm{X}$ & & & & $\mathrm{X}$ & $\mathrm{X}$ & & & 10 & 5 \\
\hline
\end{tabular}

Os trabalhos descreveram uma lista que totalizou 344 entradas de morfoespécies, mas cujas equivalências de morfoespeciação de estudos diferentes não são consistentes. Em função disso, houve necessidade de desconsiderar as morfoespécies no estudo de similaridade. Após essa triagem, foram encontradas 127 espécies com identificação final nos trabalhos. As espécies mais frequentes apresentaram repetições ao longo da faixa de distribuição de mata, foram eles: Deltochilum brasiliense, Dichotomius mormon, Eurysternus cyanescens, Coprophanaeus dardanus, Eurysternus paralellus, Phanaeus splendidulus e Eurysternus caribaeus. A análise de similaridade revelou uma agregação de grupos reconhecíveis associados à posição geográfica. Os três grupos agregam: (1) estados do sul e sudeste brasileiro, (2) estados do nordeste brasileiro e (3) amostras da Bahia e Alagoas, como pontos intermediários e similares aos grupos 1 e 3 (Figura 1). O estado com mais espécies coletadas dentro do eixo de distribuição foi Minas Gerais, seguida por São Paulo, devido grande quantidade de coletas no estado (MG) ou pela amostragem em amplas áreas preservadas, como Parques Estaduais (SP). 


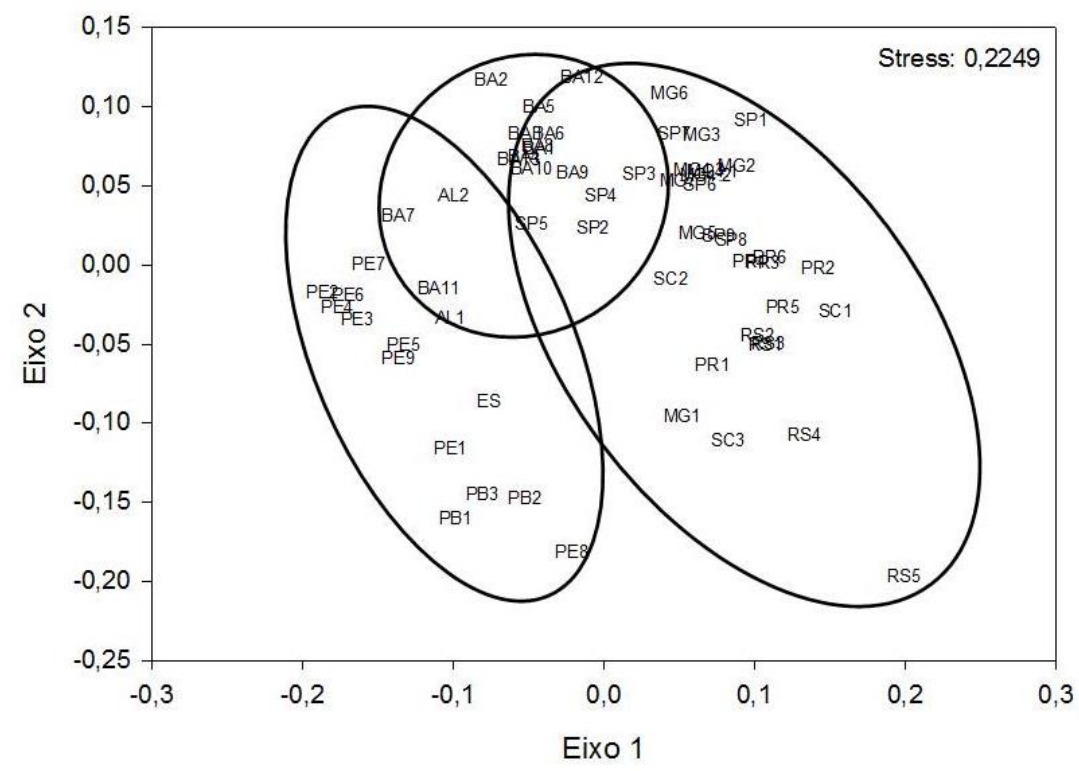

Figura 1. NMDS da similaridade (Jaccard) da distribuição de Scarabaeinae (Coleoptera, Scarabaeidae) em Mata Atlântica brasileira. As duas primeiras letras correspondem às siglas dos estados brasileiros, que estão indicados após os trabalhos listados.

Questões taxonômicas deixam dúvidas quanto à perfeita identificação de zonas de ocorrência de alta diversidade ou mesmo de maior ou menos similaridade. Há diversos trabalhos em que o número de espécies não identificadas até o nível de espécie foi maior do que as identificadas, e mesmo o registro de morfoespécies em que a proximidade ou afinidade com certos grupos de espécies (aff., prox., gr.) deixa dúvidas da amplitude da distribuição real das espécies. A aproximação do grupo de pontos na Bahia aos dois outros grupos (Nordeste/Sul+Sudeste) sugere que possa haver uma mudança da estrutura vegetal ou mesmo a interferência de outros fatores que afetam a vegetação, como o clima ou topografia. A Bahia já foi mencionada como importante área de contato entre composições vegetais do nordeste com o sudeste (Siqueira, 1994), e a fauna pode apresentar uma resposta similar, mas é questão ainda a ser estudada.

\section{CONCLUSÃO}

Com os dados apresentados, conclui-se que existe uma grande similaridade de composição entre as áreas nordestinas e entre áreas do sul e sudeste brasileiros, com uma zona de inflexão na Bahia. As áreas de alta diversidade são relacionadas às áreas amostradas de forma recorrente e a áreas preservadas, de forma que as amostras deveriam ser ampliadas nas áreas nordestinas remanescentes.

\section{REFERÊNCIAS}

HERNANDEZ, M.I.M. \& ENDRES, A.A. 2011. Besouros Escarabeídeos em Remanescentes de Mata Atlântica no Estado da Paraíba: As Espécies da Mata do Buraquinho.

SIQUEIRA, M.F. 1994. Classificação da vegetação e ordenação de espécies arbóreas da Mata Atlântica. Dissertação, Mestrado em Ecologia, Universidade Estadual de Campinas, Campinas, SP. 